\section{New Fluorescent Labeling Technologies for Ultrasensitive Cytochemical and Histochemical Imaging}

lain Johnson

Molecular Probes, Inc iain.johnson@probes.com, iainjohnson@yahoo.com

Immunofluorescence techniques using multicolor labeling and analysis of colocalization are essential for unraveling the structural and functional complexity of cells and tissues. Improvements in the capabilities of these techniques are dependent on the development of new fluorescent dyes and methods for attaching them to antibodies and other markers.

The Alexa Fluor(B) series of dyes developed by researchers at Molecular Probes, Inc. have optimized spectroscopic and physical properties that enable preparation of bioconjugates with consistently strong and photostable fluorescence output. The series consists of fourteen dyes with fluorescence excitation peaks ranging from $350 \mathrm{~nm}$ to $750 \mathrm{~nm}$. Functionally important characteristics of Alexa Fluor dyes include strong absorption at the output wavelengths of common excitation sources, resistance to photobleaching and self-quenching, and water solubility to facilitate coupling reactions with proteins and other biomolecules. The well-differentiated spectra of the fourteen Alexa Fluor dyes provide many options for multicolor labeling (FIG 1) and molecular proximity detection via fluorescence resonance energy transfer



FIG 1. A cryosection of mouse intestine showing basement membranes labeled with an anti-fibronectin antibody and Alexa Fluor 488 goat anti-chicken secondary antibody (green fluorescence). Goblet cells and crypt cells are labeled with Alexa Fluor 594 wheat germ agglutinin (red fiuorescence). The microvillar brush border and smooth muscle layer are visualized with Alexa Fluor 680 phalloidin

(FRET). In addition to their use for immunofluorescent labeling, Alexa Fluor dyes have been incorporated into a wide range of specialized probes including neuronal tracers, fluorescent lipopolysaccharides and cholera toxin $B$ conjugates for detection of lipid rafts.

Conventional multicolor immunofluorescence detection procedures require the use of primary antibodies from unrelated species of animals in conjunction with species-specific secondary antibodies, or combinations of biotin-avidin reagents with directly anti-mouse $\operatorname{lgG}$ antibody
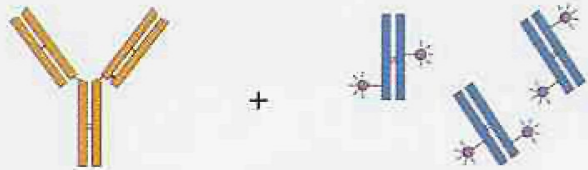

Incubate

B
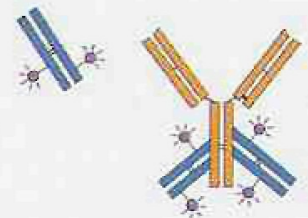

Labeled Fab fragments bound to mouse lgG
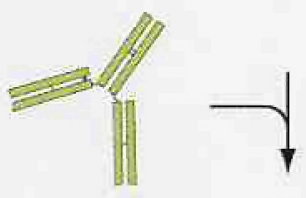

Mix with nonspecific mouse IgG

C
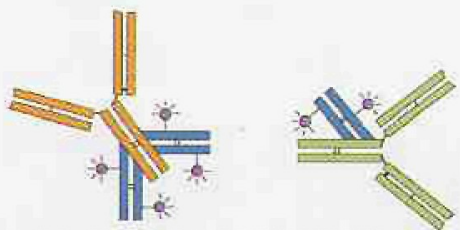

Nonspecific mouse $\lg G$ bound to excess Fab fragments

FIG 2. Schematic representation of Zenon TM labeling complex preparation. An unlabeled mouse lg $G$ is incubated with a fluorophore-labeled, Fc-specific anti-mouse Fab fragment (panel A), which binds to the Fc portion of the mouse antibody (panel B). Excess Fab fragment is adsorbed by nonspecific mouse IgG (panel $C$ ), preventing crosslabeling by the Fab fragment in experiments where multiple mouse IgG primary antibodies are present.

labeled primary and secondary antibodies. The straightforward approach of directly labeling primary antibodies has often been overlooked, primarily because chemical coupling of dyes, haptens and enzyme labels to antibodies is a moderately difficult and relatively low-yield process that is impractical for very small amounts of protein. Molecular Probes' new Zenon ${ }^{\mathrm{TM}}$ immunolabeling technology circumvents these limitations, providing a rapid, convenient and completely flexible technique for coupling dyes to antibodies. Zenon technology is based on dye-or enzyme-labeled Fab fragments of secondary antibodies directed against the $F_{c}$ regions of primary antibodies. The primary antibody types that can be recognized and detected by Zenon labeling reagents are determined by the immunospecificity of the Fab fragments from which they are prepared. Simple mixing of a Zenon labeling reagent with an intact primary antibody rapidly and quantitatively forms a labeling complex (FIG 2). Within five minutes of mixing, complex formation is complete; Zenon labeling complexes can then be used for immunodetection in the same way as covalently modified primary antibodies. For single-color immunolabeling, it is usually not necessary to remove uncomplexed Zenon labeling reagent. However, in applications that involve multiple antibodies of any type (including antibodies from other species or of other isotypes), adsorption of residual Zenon labeling reagent against nonspecific $\mathrm{lgG}$ is essential to 


\section{set your sights on a higher level}


avoid crossreactivity. Treatment with aldehyde fixatives after immunostaining permanently blocks the transfer of the labeling reagent and preserve the staining pattern.

The practical advantages of Zenon labeling technology can be summarized as follows:

- Preparation of Zenon labeling complexes for cell staining takes only five minutes. Adsorption of residual Zenon labeling reagent, which is necessary in multiplex immunolabeling applications, takes only an additional five minutes.

- A standard Zenon labeling procedure requires only $1 \mu \mathrm{g}$ of primary antibody, and even smaller quantities can be routinely labeled without sacrificing reliability and reproducibility. By comparison, conventional direct labeling typically requires $100 \mu \mathrm{g}$ of primary antibody. Zenon technology enables panels of labeled antibodies to be prepared for use in screening procedures without substantial investments of time and materials. Ultimately, it should be possible to perform automated Zenon complex preparation and immunolabeling procedures in microplates or in microfluidic devices.

- Unlike chemical labeling of antibodies, labeling of antibodies with Zenon labeling reagents does not require removal of exogenous proteins such as serum albumin from the antibody prior to complex formation.

- $100 \%$ of the primary antibody sample is labeled. The extent of antibody labeling (and thus the fluorescence intensity or enzymatic activity of Zenon labeling complexes) can be fine-tuned by variation of the molar ratio of the Zenon reagent to the primary antibody used for complex formation.

- Zenon technology permits use of multiple mouse antibodies in the same immunolabeling protocol. Furthermore, mouse primary antibodies can be used on mouse tissues without the background staining associated with the use of labeled secondary antibodies in this situation. These particular capabilities are also provided by labeled primary antibodies prepared by conventional covalent modification methods.

- Detection schemes can be optimized and adjusted by using different Zenon labeling reagents to switch from one fluorescent color to another or from dye labels to enzyme labels, without major expenditure of time and materials.

- Covalently modified primary antibodies are often partially inactivated due to indiscriminate attachment of dyes or haptens in the antigen binding site. Because Zenon labeling reagents are targeted to the $\mathrm{Fc}$ region, this problem is avoided entirely.

Enzyme-mediated detection methods permit ultrasensitive detection of low abundance targets that are below the detection threshold of other fluorescence techniques. Tyramide Signal Amplification (TSA TM) and Enzyme-Labeled Fluorescence (ELF®) are two complementary techniques that generate signal amplification based on in situ deposition of multiple fluorescent substrates per enzyme label. TSA uses horseradish peroxidase (HRP) enzyme labels and tyramide substrates, which can be coupled to a wide assortment of dyes and haptens. Higher levels of signal amplification can be achieved by detection of Oregon Green( 488 tyramide with anti-fluorescein/Oregon Green antibody conjugates labeled with HRP, followed by a second round of fluorescent tyramide deposition. The dyeanti-dye primary antibody system also provides a useful alternative to biotin-streptavidin detection in cells and tissues where the presence of endogenous biotin produces substantial background signals. ELF is based on alkaline phosphatase enzyme labels and a fluorogenic substrate that forms a yellow-green-fluorescent precipitate at the site of phosphatase activity. The precipitate is extremely photostable and exhibits an exceptionally large fluorescence Stokes shift, allowing signals to be readily discriminated from background autofluorescence and other fluorescent labels.

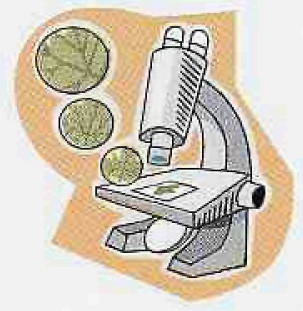

\section{POSITION AVAILABLE Electron Tomography/ Image Processing}

The Wadsworth Center seeks a motivated, responsible and mature individual to develop and implement procedures in electron tomography for federally-funded biomedical research projects. The electron microscopy facility at the Wadsworth Center is state of the art with an F20 and F30 FEI Tecnai microscopes, a JOEL 4000 with energy filter, and Zeiss 910 , all with capability for automated data collection for electron tomography. The Wadsworth Center also has an AEI 1.2 MV HVEM for thick section work, and a state of the art light microscopy facility for correlative studies. The research environment is stimulating and challenging and includes several well-funded PI's in cell and structural biology. Training will be provided for all specialized tasks.

Minimum qualifications: B.S. in physics, computer science, mathematics, chemistry, or biology; computer competence and familiarity with PC and Unix operating system. Preferred Qualifications: B.S. in physics or computer science; experience with transmission electron microscopy; experience with image processing; programming capability.

Responsibilities: Compute and analyze tomographic reconstructions for grant-funded projects; assist in developing and implementing new procedures for automating tomographic reconstruction and volume segmentation; implement and manage a database cataloging input data, project paramelers, critical procedures; results of the $3 \mathrm{D}$ reconstruction and the $3 \mathrm{D}$ analysis.
Salary commensurate with experience. Interested parties should contact:
Dr. Bruce F. McEwen
c/o Ms. Cathy Kahler
Wadsworth Center
PO Box 509
Albany, NY 12201-0509
Tel: (518) 4862882 Fax (518) 4864901
Email: ckahler@wadsworth.org 


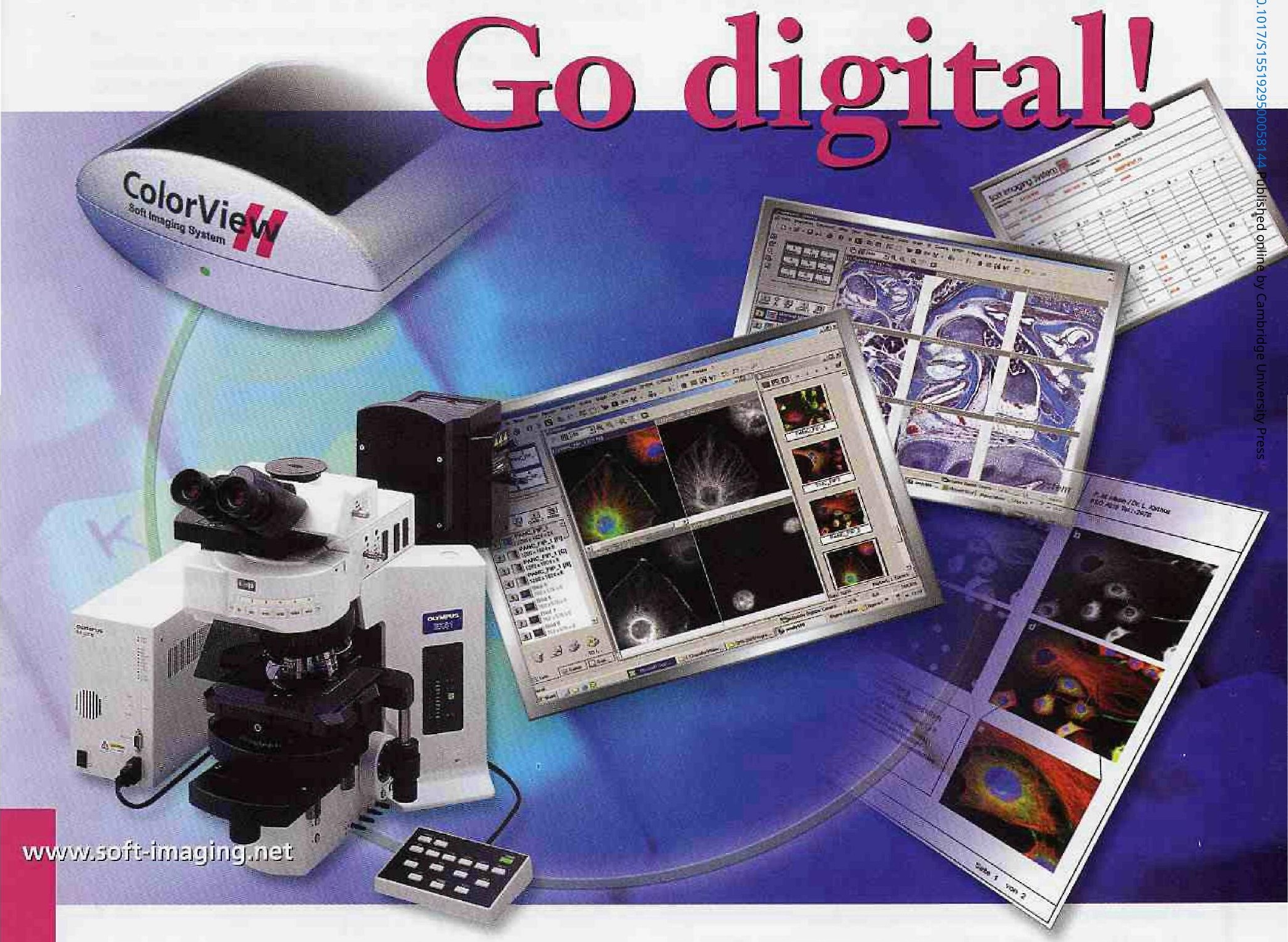

Let ofligitul inneige

analysis put you in

Whe Head

Exploit the generous scope of digital acquisition, archiving, documentation and analytical functions offered by Soft Imaging System.

The never-ending pressure to cut costs and the rising product-quality demands require efficiently-organized workflows and optimized use of device capacity.
- This is precisely what the analySIS ${ }^{\text {im }}$ image-analytical system provides you with: microscope control, camera operation, optimized image acquisition, manual or automated image analysis, database archiving, report generation, and e-mailing.

- And it's all integrated in a single software program: analySIS ${ }^{\circ}$. This saves time, reduces running costs and frees up capacity for what really is important.

For more information on analySIS ${ }^{\oplus}$ and

Soft Imaging System, visit us at:

www. soft-imaging.net
Europe

+49 (251) 798000

North America

(888) FIND SIS

$+1(303) 2349270$

Asia / Pacific +60 (3) 83181400 